DOI: $10.20472 /$ IAC.2020.053.019

IKUYA TAKAO

Aoyama Gakuin University, Japan

RYOJI SATO

Aoyama Gakuin University, Japan

TAKASHI KABURAGI

International Christian University, Japan

SATOSHI KUMAGAI

Aoyama Gakuin University, Japan

TOSHIYUKI MATSUMOTO

Aoyama Gakuin University, Japan

YOSUKE KURIHARA

Aoyama Gakuin University, Japan

\title{
DEVELOPMENT OF ONE SENSOR MULTI-PHYSICAL QUANTITIES SENSING DEVICE BASED ON PIEZOELECTRIC FILM -APPLICATION TO DETECTING OF SECURITY EVENTS-
}

\begin{abstract}
:
Generally, a sensor is specialized to measure a targeted physical quantity. Hence, to measure multi-physical quantities, the same numbers of types of sensors as physical quantities to be measured are required. However, applying plural types of sensors increases battery power consumption. In the case that a vast number of sensor nodes including plural types of sensors are spread to our living environment, a large number of broken batteries are discarded, which may lead to an environmental burden. If one sensing device can measure multi-physical quantities, the power consumption for the sensors can be saved. Therefore, we propose a sensing device that can measure multi-physical quantities (pressure, thermodynamic temperature, luminous intensity) by utilizing piezoelectric film and apply the sensing device to detect security events.

The structure of the proposed sensing device comprises an acrylic box with a port and piezoelectric film covering the port to seal the inside of the box. When a tiny pressure change is generated inside or outside of the box, the film can be distorted, thereby generating voltage corresponding to the distortion. In addition, when the box is warmed up by heat, the increased inner pressure of the box distorts the film from the inside and a voltage corresponding to the pressure is generated. Therefore, the thermodynamic temperature can be measured indirectly. In the case of the measurement of the luminous intensity, the piezoelectric film outputs voltage that is proportional to the intensity of the light irradiated to the surface of the piezoelectric film. In this study, we assume that unauthorized door opening/closing, light on/off, and fire are the security events to be detected by the sensing device. The sensing device measures the following four data types: "No abnormality" - no physical quantity detected, "Door"-that the door is opened, "Heat"-measure of thermodynamic temperature, and "Light"-measure of luminous intensity. To characterize the signal for each security event, we build a model that reconstructs a signal based on the input signal by utilizing the autoencoder. The characteristic of each security event is defined by the sum of the
\end{abstract}


root mean square error (SRMSE) that represents the similarity between the input signal and the reconstructed signal by the model. Finally, the security event is determined by applying the SRMSE to a classifier. As for the experimental results, the average error rates were 0.29 and 0.23 for the multi-class SVM classifier and k-nearest neighbors classifier, respectively.

\section{Keywords:}

Multi-Physical Quantities Sensing, Security 